Trabajos originales

\title{
Validez diagnóstica de la endosonografía biliopancreática en el diagnóstico de colangitis aguda secundaria a obstrucción biliar
}

\section{Diagnostic Validity of Biliopancreatic Endosonography in the Diagnosis of Acute Cholangitis Secondary to Biliary Obstruction}

Andrés Mauricio Ricardo R. ${ }^{1}$, Lázaro Antonio Arango M. ${ }^{2}$

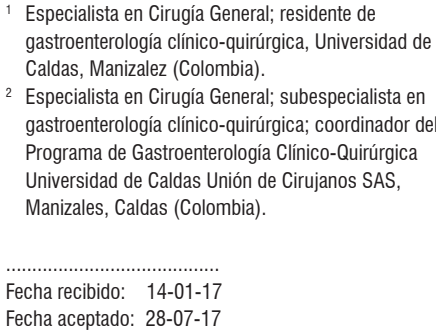

\begin{abstract}
Resumen
El diagnóstico de las colangiopatías obstructivas por endosonografía biliopancreática (EUS, por sus siglas en inglés) se ha estudiado en profundidad, y existe un extenso reconocimiento de su papel para el diagnóstico de coledocolitiasis, tumores de la encrucijada biliopancreática y síndromes de compresión extrínseca de la vía biliar. La colangitis, la complicación más frecuente de la obstrucción biliar y responsable de gran parte de su morbimortalidad, no se ha relacionado en forma suficiente con los hallazgos endosonográficos.

Nuestro estudio buscó definir la validez diagnóstica de la endosonografía en los pacientes con colangitis aguda con el fin de limitar la morbimortalidad del retraso diagnóstico. Se realizó un estudio descriptivo, analizando las historias clínicas de pacientes sometidos a endosonografía biliopancreática, por ictericia obstructiva de cualquier etiología, y quienes posteriormente fueron llevados a colangiopancreatografía retrógrada endoscópica (CPRE) para manejo de obstrucción biliar. Comparamos los hallazgos endosonográficos compatibles con colangitis aguda (engrosamiento de la vía biliar de $1,5 \mathrm{~mm}$ o más, presencia de halo pericolangítico de al menos $1,5 \mathrm{~cm}$ de longitud y presencia de contenido de ecogenicidad mixta en el interior de la vía biliar) con el drenaje purulento durante la CPRE.

Se encontró una alta frecuencia de estos hallazgos en los pacientes con colangitis, y el engrosamiento de las paredes de la vía biliar fue el más común, en el $92,6 \%$ de los casos, seguido por la presencia de halo pericolangítico en el $59,3 \%$ de los casos y contenido ductal en el $66,7 \%$ de los casos. Igualmente, se encontró asociación ascendente de las frecuencias con grado de severidad de la colangitis. Resulta recomendable realizar estudios con un mayor poder estadístico que permitan validar nuestros resultados para una futura inclusión de la EUS en el algoritmo diagnóstico de las guías de práctica clínica en colangitis aguda.
\end{abstract}

\section{Palabras clave}

Endosonografía endoscópica, colangitis, imágenes diagnósticas en colangitis, colangiopatía obstructiva, obstrucción biliar, infección biliar.

\section{INTRODUCCIÓN}

La colangitis aguda es la complicación más frecuente de la obstrucción biliar, con una mortalidad de hasta el $10 \%$ en quienes el drenaje de la vía biliar no se hace tempranamente (1). La clínica en forma temprana solo se presenta en el $26,4 \%$ de los casos, por lo que las guías internacionales recomiendan criterios complejos que adicionan a la clínica hallazgos de laboratorio e imágenes diagnósticas, con una sensibilidad que no alcanza el $90 \%$ y una especificidad inferior al $80 \%(2)$.

La piedra angular de la terapia de la colangitis aguda es la descompresión y el drenaje biliar tempranos. La presencia de material purulento en el drenaje es un signo patognomónico de colangitis aguda, y la CPRE es el mejor método para el drenaje biliar, con un éxito del $98 \%$ y con menores 


\begin{abstract}
Diagnosis of obstructive cholangiopathy with biliopancreatic endosonography (BUS) has been extensively studied, and its role in the diagnosis of choledocholithiasis, biliopancreatic junction tumors and extrinsic biliary compression syndromes is widely recognized. Endosonographic diagnosis of cholangitis, a more frequent complication of biliary obstruction which is responsible for much of its morbidity and mortality, has not been sufficiently studied.

Our study's objective is to define the diagnostic validity of endosonography for patients with acute cholangitis in order to limit morbidity and mortality due to diagnostic delay.

This is a descriptive study that analyzes clinical histories of patients who underwent biliopancreatic endosonography because of obstructive jaundice of any etiology and who later underwent endoscopic retrograde cholangiopancreatography (ERCP) to manage biliary obstruction. We compared endosonographic findings compatible with acute cholangitis: thickening of the bile duct of $1.5 \mathrm{~mm}$ or more, periportal halo sign of at least $1.5 \mathrm{~cm}$ in length, mixed echogenicity of bile duct content, and purulent drainage during ERCP.

Patients with cholangitis most frequently presented thickened gallbladders ( $92.6 \%$ of cases) periportal halo signs (59.3\% of cases) and mixed echogenicity of bile duct content $(66.7 \%$ of cases). We also found an ascending association of frequencies with the degree of severity of cholangitis.

Studies with greater statistical power are needed for validation of our results and for future inclusion of EUS in the diagnostic algorithm for clinical practice guidelines in cases of acute cholangitis.
\end{abstract}

\title{
Keywords
}

Endoscopic endosonography, cholangitis, diagnostic imaging of cholangitis, obstructive cholangiopathy, biliary obstruction, biliary infection.

tasas de complicaciones en comparación con la cirugía (3, 4). La evidencia reciente sugiere que resulta ideal, para reducir la morbimortalidad, realizar el drenaje biliar en las primeras 24 horas en pacientes con colangitis severa y un plazo máximo de 48 horas para los pacientes con colangitis leve a moderada (1).

La EUS biliopancreática permite explorar casi el 100\% de la vía biliar $(9,10,11)$ y, en detección de coledocolitiasis, ha demostrado tener la misma sensibilidad que la CPRE ( 8 , 12). Igualmente, ha mostrado su valor en detección de cáncer pancreático con una sensibilidad del $93 \%$ al 100\%, de tumores del conducto biliar con una sensibilidad del $80 \%$ $(12,13)$ y de estenosis biliares benignas $(14,16,17)$.

En colangitis aguda, solo un estudio basa su interés en el papel diagnóstico de la endosonografia radial en colangitis aguda. Dicho estudio encontró que el engrosamiento de la pared $>1,5 \mathrm{~mm}$, el cambio de la ecogenicidad pericoledociana $>15 \mathrm{~mm}$ de longitud y el contenido ecogénico luminal con o sin sombra acústica fueron los hallazgos endosonográficos más relevantes, los cuales son altamente predictivos de coledocolitiasis y permiten un drenaje biliar temprano, por CPRE, en las primeras 12 horas subsiguientes al diagnóstico (15).

Nuestro estudio buscó encontrar la asociación entre estos cambios endosonográficos descritos y la presencia de colangitis aguda evidenciada en el drenaje biliar por CPRE, así como la posible asociación de los hallazgos con los diferentes grados de severidad de la colangitis y su posible implicación pronóstica.

\section{MATERIALES Y MÉTODOS}

Se desarrolló un estudio descriptivo, retrospectivo, observacional y analítico cuyo objetivo fue determinar el valor predictivo positivo de la endosonografía en el diagnóstico de colangitis aguda determinado por CPRE. De igual forma, buscamos caracterizar - por etiología, severidad de la colangitis, edad y sexo- los hallazgos endosonográficos en nuestros pacientes entre abril y octubre de 2016, en Unión de Cirujanos SAS, Clínica de La Presentación, en Manizales (Colombia).

El proyecto se presentó ante el comité de postgrados y bioética de la Universidad de Caldas, donde fue aprobado y se confirmó que se correspondía con las normas éticas propuestas por la Declaración de Helsinki modificadas para el año 2005, con lo cual se procedió a la recolección de datos por medio de un instrumento previamente establecido y aprobado por el comité.

Los datos fueron extraídos de la historia clínica de pacientes llevados a endosonografía pancreatobiliar por obstrucción biliar y posterior CPRE para resolución de dicha afección, pero se excluyeron las historias clínicas con datos insuficientes respecto de la presencia de cambios endosonográficos de colangitis o del tipo de drenaje obtenido en la CPRE.

Los datos se procesaron con el software estadístico SPSS Statistics versión 23 de IBM, y la categorización de las variables se diseñó con el objeto de responder a los objetivos expresados. Las variables demográficas fueron el sexo y la edad, 
la cual se dicotomizó por encima y por debajo de 75 años, siguiendo la sugerencia de las guías de Tokio con respecto al incremento del riesgo por encima de esta edad. Dentro de las causas de colangitis, utilizamos las más frecuentes en nuestro medio. En este caso, no se tomaron en cuenta las causas iatrogénicas debido a su infrecuencia, ya que no se presentaron durante el tiempo del estudio. Para las variables de severidad, se siguió la clasificación de Tokio de 2013.

En cuanto a la presencia de hallazgos endosonográficos, se utilizaron los descritos en la literatura con mayor frecuencia y previamente comentados aquí, como variables independientes. Se realizó el análisis en tablas de 2 × 2 , además de pruebas del análisis estadístico del Chi cuadrado y la prueba exacta de Fisher, para determinar la asociación en muestras pequeñas, como es el caso de este estudio.

\section{RESULTADOS}

Se evaluó una muestra de 30 pacientes que cumplieron los criterios de inclusión. Debido a lo limitado de la muestra, se observó heterogeneidad en las variables demográficas, con una mayor frecuencia del sexo femenino ( $70 \%$ de mujeres frente a $30 \%$ de hombres).

Las causas de obstrucción biliar fueron diversas: la más frecuente de ellas correspondió a litiasis de la vía biliar en el $63,3 \%$ de los casos, seguida por lesiones malignas de la encrucijada biliopancreática en el $16,7 \%$, estenosis de etiología benigna en el 13,3\% y dos casos de obstrucción por compresión extrínseca de la vía biliar que correspondieron al 6,7\%, en un paciente de cada sexo. Discriminadas por sexo, las causas de obstrucción biliar presentaron un comportamiento similar al de la muestra global: la litiasis de la vía biliar se presentó un 55,6\% de los casos de obstrucción biliar en los hombres y en un $66,7 \%$ en las mujeres; las estenosis malignas se presentaron en un $11,1 \%$ en los hombres y en un $19 \%$ en las mujeres, aunque, en este caso, de los 5 pacientes con causa maligna de ictericia, el $80 \%$ fueron mujeres, lo que no se corresponde con el comportamiento histórico de las neoplasias de la encrucijada biliopancreática, que suelen ser de mayor frecuencia en el género masculino, aunque se explica por el limitado tamaño de la muestra.

En cuanto a estenosis benigna, para los pacientes masculinos fue la causa de obstrucción biliar en el $22,2 \%$ de los casos, y solo en el 9,5\% de las mujeres y, en cuanto a la compresión extrínseca, si bien se presentó un caso en cada sexo, este correspondió en los hombres al 11\% de las causas de obstrucción biliar y solo al 4,8\% de ellas en las mujeres (Tabla 1). Cuando se analizaron las causas según la edad dicotomizada, en función de la edad de riesgo según las guías de Tokio de 2013, en mayores y menores de 75 años, encontramos que la mayoría de los casos se ubican en un rango de edad inferior a los 75 años $-56,7 \%$ de los casos-, sin encontrar diferencias estadísticamente significativas, con una $\mathrm{p}$ de 0,64 .

Tabla 1. Frecuencias etiológicas de la obstrucción biliar según el sexo.

\begin{tabular}{lcc}
\hline & \multicolumn{2}{c}{ Sexo del paciente según historia clínica } \\
\cline { 2 - 3 } & Masculino & Femenino \\
\hline Litiasis & $26,3 \%$ & $73,7 \%$ \\
Estenosis maligna & $20,0 \%$ & $80,0 \%$ \\
Estenosis benigna & $50,0 \%$ & $50,0 \%$ \\
Compresión extrínseca & $50,0 \%$ & $50,0 \%$ \\
\hline
\end{tabular}

Causas de obstrucción biliar en el servicio de Gastroenterología de la Unión de Cirujanos SAS, disciminadas por sexo.

Igualmente, se demostró que, para ambos rangos de edad, la principal causa de obstrucción biliar resultó ser la litiasis: el 70,6\% de las causas en los menores de 75 años y el 53,8\% en los mayores. Curiosamente, el 17,6\% de los menores de 75 años presentaron estenosis maligna, lo que representa un porcentaje superior que en el grupo de mayor edad (solo el 15,4\%). La evaluación de la estenosis maligna y la compresión extrínsec, resulta problemática debido a que, en el primer caso, no se presentaron pacientes menores de 75 años y, en el caso de la compresión extrínseca, los dos pacientes que mostraron este diagnóstico eran menores de 75 años, por lo que la interpretación de los resultados resulta difícil (Tabla 2).

Tabla 2. Frecuencias etiológicas de la obstrucción biliar según la edad dicotomizada por riesgo (tabla cruzada).

\begin{tabular}{|c|c|c|c|c|c|}
\hline & & & \multicolumn{2}{|c|}{ Edad dicotomizada } & \multirow[b]{2}{*}{ Total } \\
\hline & & & $\begin{array}{c}\text { Menores } \\
\text { de } 75 \\
\text { años }\end{array}$ & $\begin{array}{c}\text { Mayores } \\
\text { de } 75 \\
\text { años }\end{array}$ & \\
\hline \multirow{10}{*}{$\begin{array}{l}\text { Causa de la } \\
\text { obstrucción } \\
\text { de la vía } \\
\text { biliar por } \\
\text { diagnóstico } \\
\text { final }\end{array}$} & Litiasis & $\mathrm{n}$ & 12 & 7 & 19 \\
\hline & & $\%$ & $70,6 \%$ & $53,8 \%$ & $63,3 \%$ \\
\hline & Estenosis & $\mathrm{n}$ & 3 & 2 & 5 \\
\hline & maligna & $\%$ & $17,6 \%$ & $15,4 \%$ & $16,7 \%$ \\
\hline & Estenosis & $\mathrm{n}$ & 0 & 4 & 4 \\
\hline & benigna & $\%$ & $0,0 \%$ & $30,8 \%$ & $13,3 \%$ \\
\hline & Compresión & $\mathrm{n}$ & 2 & 0 & 2 \\
\hline & extrínseca & $\%$ & $11,8 \%$ & $0,0 \%$ & $6,7 \%$ \\
\hline & Total & $\mathrm{n}$ & 17 & 13 & 30 \\
\hline & & $\%$ & $100,0 \%$ & $100,0 \%$ & $100,0 \%$ \\
\hline
\end{tabular}

Causas de obstrucción biliar en el servicio de Gastroenterología Unión de Cirujanos SAS, discriminadas por edad de los pacientes dicotomizada en mayores y menores de 75 años, edad en la cual aumenta el riesgo de severidad según las guías de Tokio de 2013. 
Se analizaron los hallazgos endosonográficos asociados con colangitis aguda en todos los pacientes y se caracterizaron según el sexo del paciente y los grupos etarios de interés, con el fin de definir si existía alguna asociación de los cambios endosonográficos con estas características. En ninguno de los casos se encontró asociación de las variables demográficas con los cambios endosonográficos. Igualmente, se logró determinar que las frecuencias, diferenciadas para cada hallazgo endosonográfico, no variaron en forma constante ni estadísticamente significativa respecto de la edad o el sexo de los pacientes, con lo cual se puede establecer que son variables independientes en el diagnóstico endosonográfico de la colangitis aguda.

El análisis de cada hallazgo endosonográfico por separado evidenció que el engrosamiento de las paredes de la vía biliar igual o >1,5 mm se encontró en el 89,3\% de todos los pacientes positivos en la CPRE. Solo el 7,4\% no presentó engrosamiento biliar en la endosonografía. Solo 3 pacientes no presentaron el diagnóstico final en CPRE (10\% de la muestra), pero ninguno de estos pacientes presentó engrosamiento de la pared de la vía biliar (Tablas 3 y 4 ).

Tabla 3. Frecuencia del engrosamiento de la vía biliar $>1,5 \mathrm{~mm}$ en pacientes con colangitis aguda evidenciada en CPRE (tabla cruzada).

\begin{tabular}{|c|c|c|c|c|c|}
\hline & & & \multicolumn{2}{|c|}{$\begin{array}{l}\text { Diagnóstico final de } \\
\text { colangitis por CPRE }\end{array}$} & \multirow[t]{2}{*}{ Total } \\
\hline & & & Sí & No & \\
\hline \multirow{4}{*}{$\begin{array}{l}\text { Engrosamiento de la } \\
\text { pared de la vía biliar } \\
\text { por EUS }>1,5 \mathrm{~mm}\end{array}$} & & $\mathrm{n}$ & 25 & 3 & 28 \\
\hline & $\mathrm{SI}$ & $\%$ & $89,3 \%$ & $10,7 \%$ & $100,0 \%$ \\
\hline & $\mathrm{Nl}$ & $\mathrm{n}$ & 2 & 0 & 2 \\
\hline & ino & $\%$ & $100,0 \%$ & $0,0 \%$ & $100,0 \%$ \\
\hline \multirow{2}{*}{\multicolumn{2}{|c|}{ Total }} & $n$ & 27 & 3 & 30 \\
\hline & & $\%$ & $90,0 \%$ & $10,0 \%$ & $100,0 \%$ \\
\hline
\end{tabular}

Coincidencia del hallazgo endosonográfico de engrosamiento de la pared biliar con el hallazgo en CPRE de drenaje purulento, lo cual evidencia una frecuencia de posibilidad en el $89,3 \%$ de los casos.

Aunque estos hallazgos nos sugieren que la presencia de engrosamiento la pared de la vía biliar en la endosonografia tiende a presentarse en la gran mayoría de los casos de colangitis confirmada en CPRE, con un valor predictivo del $90 \%$ calculado para este único hallazgo, la aplicación de pruebas estadísticas falla al encontrar asociación con un p de 0,626. La presencia de halo pericolangítico $>1,5 \mathrm{~cm}$ fue menos común y se encontró en el 59,3\% de los pacientes diagnosticados con colangitis por CPRE, y la mayoría de estos pacientes con halo endosonográfico $(88,9 \%)$ presentaron el diagnóstico final de colangitis aguda (Tablas 5 y 6).
Tabla 4. Frecuencia de diagnóstico de colangitis aguda en pacientes con engrosamiento de la vía biliar por endosonografía (tabla cruzada).

\begin{tabular}{lccccc}
\hline & & \multicolumn{2}{c}{$\begin{array}{c}\text { Engrosamiento de la pared de la } \\
\text { vía biliar por EUS }>\mathbf{1 , 5} \mathbf{~ m m}\end{array}$} & Total \\
\cline { 3 - 4 } & & \multicolumn{2}{c}{ Sí } & No & \\
\hline Diagnóstico & Sí & $\mathrm{n}$ & 25 & 0 & 27 \\
final de & $\%$ & $92,6 \%$ & $7,4 \%$ & $90 \%$ \\
colangitis & & $\mathrm{n}$ & 3 & 0 & 3 \\
por CPRE & No & $\%$ & $100,0 \%$ & $0,0 \%$ & $10 \%$ \\
\multicolumn{1}{r}{ Total } & $\mathrm{n}$ & 28 & 3 & 30 \\
& $\%$ & $93,3 \%$ & $6,7 \%$ & $100,0 \%$ \\
\hline
\end{tabular}

Se encontró drenaje purulento durante la CPRE en el 92,6\% de los pacientes. Tres pacientes presentaron un hallazgo endosonográfico falso positivo, lo que corresponde al $10 \%$ de la muestra.

Tabla 5. Frecuencia de halo pericolangítico $>1,5 \mathrm{~cm}$ en pacientes con colangitis aguda evidenciada en CPRE (tabla cruzada).

\begin{tabular}{lccccc}
\hline & & \multicolumn{2}{c}{$\begin{array}{c}\text { Diagnóstico final de } \\
\text { colangitis por CPRE }\end{array}$} & Total \\
\cline { 3 - 5 } & & \multicolumn{2}{c}{ Sí } & No & \\
\hline Presencia de & Sí & $\mathrm{n}$ & 16 & 2 & 18 \\
halo hipoecoico & & $\%$ & $59,3 \%$ & $66,7 \%$ & $60,0 \%$ \\
pericolangítico de & No & $\mathrm{n}$ & 11 & 1 & 12 \\
$1,5 \mathrm{~cm}$ en EUS & No & $\%$ & $40,7 \%$ & $33,3 \%$ & $40,0 \%$ \\
\multicolumn{1}{c}{ Total } & $\mathrm{n}$ & 27 & 3 & 30 \\
& & $\%$ & $100,0 \%$ & $100,0 \%$ & $100,0 \%$ \\
\hline
\end{tabular}

El halo pericolangítico se encontró en el 59,3\% de los pacientes con drenaje purulento de la via biliar en CPRE. Dos tercios de los pacientes negativos para colangitis en CPRE también presentaron el hallazgo, pero cabe destacar el escaso número de pacientes negativos en CPRE para colangitis.

Tabla 6. Frecuencia de diagnóstico de colangitis aguda en pacientes con halo pericolangítico en endosonografía (tabla cruzada).

\begin{tabular}{lccccc}
\hline & & \multicolumn{2}{c}{$\begin{array}{c}\text { Presencia de halo hipoecoico } \\
\text { pericolangítico de } \mathbf{1 , 5} \text { cm en EUS }\end{array}$} & Total \\
\cline { 3 - 5 } & & \multicolumn{2}{c}{ Sí } & No & \\
\hline Diagnóstico & Sín & $n$ & 16 & 11 & 27 \\
final de & $\%$ & $88,9 \%$ & $91,7 \%$ & $90,0 \%$ \\
colangitis & & $n$ & 2 & 1 & 3 \\
por CPRE & No & $\%$ & $11,1 \%$ & $8,3 \%$ & $10,0 \%$ \\
\multicolumn{1}{c}{ Total } & $\mathrm{n}$ & 18 & 12 & 30 \\
& $\%$ & $100 \%$ & $100,0 \%$ & $100,0 \%$ \\
\hline
\end{tabular}

Se encontró drenaje purulento durante la CPRE en el 88,9\% de los pacientes con halo endosonográfico. El drenaje purulento se encontró igualmente en una alta proporción de pacientes negativos para este hallazgo endosonográfico. No se evidenció significancia estadística. 
El contenido intraductal en la endosonografía y el diagnóstico final de colangitis se evidenciaron en el 63\% de los casos. Sin embargo, cuando el hallazgo endosonográfico fue positivo, el $94,7 \%$ de estos pacientes tenían colangitis aguda. Nuevamente, encontramos la dificultad del bajo número de casos totales y del escaso número de pacientes sin colangitis en el diagnóstico final, lo que no nos permite hacer un cálculo adecuado del valor predictivo del hallazgo. Once pacientes no presentaban contenido intraductal en la endosonografía, pero el $81,8 \%$ presentaron colangitis aguda, lo que muestra una tendencia de baja sensibilidad que requeriría una muestra mayor para poder demostrarse.

La evaluación endosonográfica respecto de la severidad según los criterios de Tokio mostró que el engrosamiento de la vía biliar estuvo presente en todos los pacientes con colangitis severa, en el $93,8 \%$ de los pacientes con colangitis moderada y en el $90,9 \%$ de las colangitis leves, con una p no significativa de 0,851 (Tablas 7 y 8 ). El halo pericolangítico estuvo presente en el $100 \%$ de los pacientes con colangitis de grado III, en el 50\% de colangitis de grado II y en el $36,6 \%$ de las colangitis de grado I, con un valor de $\mathrm{p}$ no significativo de 0,256 (Tabla 9).

Tabla 7. Severidad de la colangitis aguda en engrosamiento edosonográfico de la vía biliar (tabla cruzada).

\begin{tabular}{|c|c|c|c|c|c|}
\hline & & & \multicolumn{2}{|c|}{$\begin{array}{c}\text { Engrosamiento de la } \\
\text { pared de la vía biliar } \\
\text { por EUS }>1,5 \mathrm{~mm}\end{array}$} & \multirow[t]{2}{*}{ Total } \\
\hline & & & Sí & No & \\
\hline \multirow{6}{*}{$\begin{array}{l}\text { Severidad de } \\
\text { la colangitis } \\
\text { según la } \\
\text { clínica del } \\
\text { paciente }\end{array}$} & \multirow{2}{*}{$\begin{array}{l}\text { Grado III: falla } \\
\text { de órgano }\end{array}$} & $n$ & 3 & 0 & 3 \\
\hline & & $\%$ & $10,7 \%$ & $0,0 \%$ & $10,0 \%$ \\
\hline & \multirow{2}{*}{$\begin{array}{l}\text { Grado II: } \\
\text { respuesta } \\
\text { inflamatoria } \\
\text { sistémica }\end{array}$} & $n$ & 15 & 1 & 16 \\
\hline & & $\%$ & $53,6 \%$ & $50,0 \%$ & $53,3 \%$ \\
\hline & \multirow{2}{*}{$\begin{array}{l}\text { Grado I: sin } \\
\text { respuesta } \\
\text { inflamatoria ni } \\
\text { falla de órgano }\end{array}$} & $n$ & 10 & 1 & 11 \\
\hline & & $\%$ & $35,7 \%$ & $50,0 \%$ & $36,7 \%$ \\
\hline \multirow{2}{*}{\multicolumn{2}{|c|}{ Total }} & $\mathrm{n}$ & 28 & 2 & 30 \\
\hline & & $\%$ & $100,0 \%$ & $100,0 \%$ & $100,0 \%$ \\
\hline
\end{tabular}

Se evidenció engrosamiento de la vía biliar en los pacientes con colangitis severa, lo que corresponde al $10,7 \%$ de la muestra. La mayoría de los pacientes presentaron una severidad moderada, con una alta frecuencia de engrosamiento endosonográfico de la vía biliar en estos casos.

El contenido intraductal, mostró igual comportamiento en colangitis severa y se presentó solo en el $68 \%$ de los pacientes con colangitis de grado II y en el $45 \%$ de los pacientes con colangitis leve. La búsqueda de asociación en las frecuencias de las diversas causas no demostró signifi- cancia ni una tendencia a mayor positividad de los hallazgos con respecto a la etiología.

Tabla 8. Frecuencia del engrosamiento endosonográfico de la vía biliar en función de la severidad clínica, según las guías de Tokio de 2013 (tabla cruzada).

\begin{tabular}{|c|c|c|c|c|c|c|}
\hline & \multicolumn{3}{|c|}{ Severidad de la colangitis } & \multirow[t]{2}{*}{ Total } \\
\hline & & & Grado III & Grado II & Grado I & \\
\hline \multirow{5}{*}{$\begin{array}{l}\text { Engrosamiento } \\
\text { de la pared de la } \\
\text { vía biliar por EUS } \\
>1,5 \mathrm{~mm}\end{array}$} & \multirow{2}{*}{ Sí } & $\mathrm{n}$ & 3 & 15 & 10 & 28 \\
\hline & & $\%$ & $100,0 \%$ & $93,8 \%$ & $90,9 \%$ & $93,3 \%$ \\
\hline & \multirow{3}{*}{ No } & $n$ & 0 & 1 & 1 & 2 \\
\hline & & $\%$ & $0,0 \%$ & $6,3 \%$ & $9,1 \%$ & $6,7 \%$ \\
\hline & & $\mathrm{n}$ & 3 & 16 & 11 & 30 \\
\hline Total & & $\%$ & $100 \%$ & $100 \%$ & $100,0 \%$ & $100,0 \%$ \\
\hline
\end{tabular}

Se encontró engrosamiento biliar en el $100 \%$ de los pacientes con colangitis severa, 93,8\% de colangitis moderada y $90,9 \%$ de colangitis leve, lo cual sugiere una mayor coincidencia del hallazgo con grados mayores de colangitis y una posible utilidad pronóstica que requerirá nuevos estudios para demostrar significancia estadística.

Tabla 9. Frecuencia del halo pericolangítico en endosonografia de la vía biliar en función de la severidad clínica, según las guías de Tokio de 2013 (tabla cruzada).

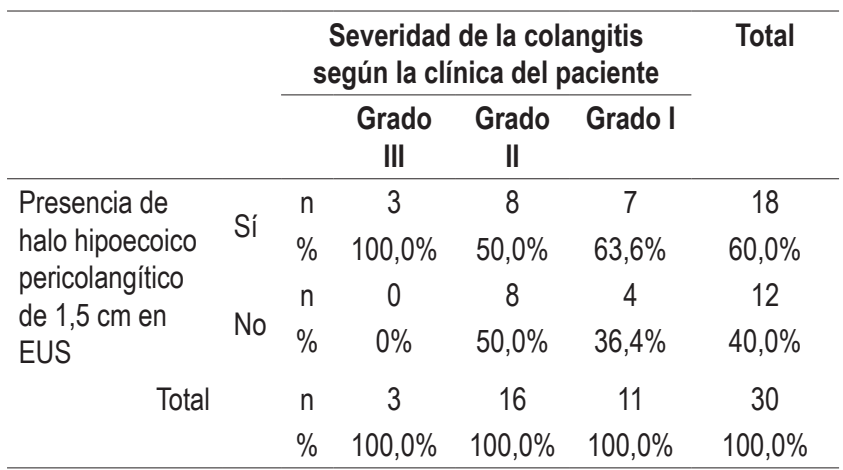

La presencia de halo endosonográfico presentó un comportamiento similar, ya que estuvo presente en todos los pacientes con colangitis severa, en el $50 \%$ de las colangitis moderadas y en el 63,6\% de las colangitis severas.

\section{DISCUSIÓN}

Establecer un diagnóstico más preciso de la colangitis aguda implica una posibilidad de disminuir el lapso entre el diagnóstico y el tratamiento de una enfermedad con alta mortalidad y que se incrementa en forma prohibitiva conforme el tratamiento se atrasa (5). Boender y colaboradores encontraron un $67 \%$ de mortalidad frente a un $1 \%$ si el tiempo transcurrido hasta el drenaje biliar es de 72 horas $(6,7)$. El presente estudio buscó encontrar la validez diag- 
nóstica de la endosonografia biliopancreática lineal en el diagnóstico de colangitis aguda.

Se encontró que el engrosamiento concéntrico de la vía biliar superior a 1,5 mm está presente en el $89,3 \%$ de los casos de colangitis aguda, con un valor predictivo positivo del $90 \%$. De igual manera, ante la presencia de halo pericolangítico, la colangitis se confirmó en el $88,9 \%$ de los casos $y$, en el caso de contenido intraductal, en el $94,7 \%$ de ellos.

Estos resultados, aunque prometedores, no mostraron validez estadística en los diferentes cálculos de asociación, posiblemente debido a lo pequeño de la muestra recolectada y a que no se controló la muestra con un grupo homogéneo de pacientes con colestasis pero sin diagnóstico clínico de colangitis, lo que, posiblemente, hubiera permitido un cálculo preciso de los valores predictivos y la obtención de una muestra representativa, como se encontró en el estudio de Emrah y colaboradores. En dicho estudio, los autores describieron características endosonográficas iguales, comparadas con un grupo control de pacientes que no presentan la afección, y encontraron engrosamiento de la pared en el $68 \%$ de los casos, halo en el $46 \%$ y contenido ductal de ecos mixtos en el 64\%. Estos datos responden a medidas de asociación con $\mathrm{p}$ inferior al 0,05 para la presencia de engrosamiento de la vía biliar, pero sin asociación medible en los otros dos parámetros (15).

Ambos estudios muestran una tendencia con respecto a la capacidad diagnóstica de la endosonografía para realizar un diagnóstico precoz de colangitis, pero, a pesar de que, en ambos, el engrosamiento de la pared de la vía biliar se muestra como el hallazgo de mayor importancia, no es posible en ninguno de los dos estudios determinar una precisión mayor de la de los criterios de diagnóstico actuales, posiblemente debido a sesgos metodológicos dentro de ambos trabajos y al bajo número de pacientes estudiados.

\section{CONCLUSIONES}

La colangitis aguda es una patología de prevalencia alta y de morbimortalidad importante a pesar del manejo antibiótico. Los métodos endosonográficos no han sido evaluados lo suficiente en cuanto al diagnóstico de colangitis, y se desconoce con certeza su desempeño en este punto a pesar de la gran resolución de las estructuras biliopancreáticas y de su mejor desempeño diagnóstico en gran parte de las patologías estructurales a este nivel.

El presente estudio no logró obtener resultados de asociación que permitieran extrapolar datos a la población general, pero se ha logrado obtener datos de tendencia, con alta frecuencia de hallazgos endosonográficos que sugieren colangitis aguda cuando esta se confirma por el drenaje purulento en CPRE. Dentro de los hallazgos endosonográficos, el de mayor valor para este estudio es la presencia de engrosamiento de la vía biliar, la cual se encontró en la mayoría de los pacientes con colangitis aguda.

Otros hallazgos son el halo pericolangítico y la presencia de contenido hipoecogénico en la vía biliar, que se encontraron con frecuencias más variables en el estudio, pero siempre con una tendencia mayor hacia los pacientes con confirmación diagnóstica. Sin embargo, sobre estos hallazgos, hay menor claridad.

Igualmente relevante resulta el hallazgo relacionado con la mayor frecuencia de cada uno de los cambios endosonográficos en relación con la severidad, ya que, en todos los casos, se demostró una mayor frecuencia en la medida en que esta aumenta. Esto es de capital importancia, ya que implica la posibilidad de un papel no solo diagnóstico, sino también pronóstico de la endosonografía en la colangitis aguda, con modificaciones adicionales en el tiempo de la terapia, que podrían disminuir la morbimortalidad a futuro.

Por ahora, se deberá recomendar la realización de estudios adicionales que nos permitan obtener un mayor poder estadístico y conclusiones que se puedan aplicar en las guías clínicas. Sería ampliamente recomendable para futuros estudios obtener una ampliación de la muestra, con un adecuado cálculo de ella y la comparación con controles sanos para lograr el poder estadístico deseado.

Igualmente, es recomendable, en el ámbito clínico, que en todos los estudios endosonográficos por obstrucción biliar clínica, se tengan en cuenta la medición de la pared de la vía biliar, la presencia de halo pericolangítico y el contenido intraductal mixto, y se proceda en consecuencia a estos hallazgos para acelerar la implementación de medidas adecuadas para el drenaje de la vía biliar y la disminución de la morbimortalidad asociada con la colangitis, ya que, si bien no existe evidencia fuerte, la tendencia y el potencial beneficio exceden el riesgo de acelerar medidas que, en definitiva, requerirá el paciente para el manejo de su enfermedad de base.

\section{Agradecimientos}

Al Dr. Lázaro Antonio Arango M. por su apoyo denodado al estudio al contribuir con la asesoría académica y facilitar el acceso a las historias clínicas para la recolección de datos. A la Dra. Luz Elena Sepúlveda, asesora metodológica del estudio. A Unión de Cirujanos SAS, institución que, por el alto volumen y como centro de referencia regional y nacional, nos permitió acceder a sus archivos clínicos. A la Universidad de Caldas, institución que avala y cualifica el presente estudio. 


\section{REFERENCIAS}

1. TG13: Updated Tokyo Guidelines for acute cholangitis and acute cholecystitis. J Hepatobiliary Pancreat Sci. 2013:20:24-34.

2. Kochar R, Banerjee S. Infections of the biliary tract. Gastrointest Endoscopy Clin N Am. 2013;23:199-218. Doi: https://doi.org/10.1016/j.giec.2012.12.008

3. Kinney TP. Management of ascending cholangitis. Gastrointest Endosc Clin N Am. 2007;17(2):289-306. Doi: https://doi.org/10.1016/j.giec.2007.03.006

4. Cohen S, Bacon BR, Berlin JA, et al. National Institutes of Health State of the Science Conference Statement: ERCP for diagnosis and therapy, January 14-16, 2002. Gastrointest Endosc. 2002;56(6):803-9. Doi: https://doi.org/10.1016/ S0016-5107(02)70351-9 / https://doi.org/10.1067/ mge.2002.129875

5. Welch JP, Donaldson GA. The urgency of diagnosis and surgical treatment of acute suppurative cholangitis. Am J Surg. 1976;131(5):527-32. Doi: https://doi.org/10.1016/00029610(76)90003-9

6. O’Connor MJ, Schwartz ML, McQuarrie DG, et al. Acute bacterial cholangitis: an analysis of clinical manifestation. Arch Surg. 1982;117(4):437-41. Doi: https://doi. org/10.1001/archsurg.1982.01380280031007

7. Boender J, Nix GA, de Ridder MA, et al. Endoscopic sphincterotomy and biliary drainage in patients with cholangitis due to common bile duct stones. Am J Gastroenterol. 1995;90(2):233-8

8. Chak A, Cooper GS, Lloyd LE, et al. Effectiveness of ERCP in cholangitis: a community-based study. Gastrointest Endosc. 2000;52(4):484-9. Doi: https://doi.org/10.1067/ mge.2000.108410
9. Verma D, Kapadia A, Eisen GM, et al. EUS vs MRCP for detection of choledocholithiasis. Gastrointest Endosc. 2006;64:24854. Doi: https://doi.org/10.1016/j.gie.2005.12.038

10. DiMagno EP, Buxton JL, Regan PT, et al. Ultrasonic endoscope. Lancet. 1980;1:629-31. Doi: https://doi. org/10.1016/S0140-6736(80)91122-8

11. Mohamed AM, Wael AA. Endoscopic ultrasound in gastroenterology: from diagnosis to therapeutic implications. World J Gastroenterol. 2014;20(24):7801-7. Doi: https:// doi.org/10.3748/wjg.v20.i24.7801

12. Harinck F, Bruno MJ. Endosonography in the management of biliopancreatic disorders. Best Practice \& Research Clinical Gastroenterology. 2009;23:703-10. Doi: https:// doi.org/10.1016/j.bpg.2009.06.008

13. Legmann P, Vignaux O, Dousset B, et al. Pancreatic tumors: comparison of dual-phase helical CT and endoscopic sonography. Am J Roentgenol. 1998;170:1315-22. Doi: https:// doi.org/10.2214/ajr.170.5.9574609

14. DeWitt J, Misra VL, Leblanc JK, et al. EUS-guided FNA of proximal biliary strictures after negative ERCP brush cytology results. Gastrointest Endosc. 2006;64(3):325-33. Doi: https://doi.org/10.1016/j.gie.2005.11.064

15. Alper E, Unsal B, Buyraç Z, et al. Role of radial endosonography in the diagnosis of acute cholangitis. Digestive Diseases and Sciences. 2011;56(7):2191-6. Doi: https:// doi.org/10.1007/s10620-010-1552-3

16. Şurlin V, Săftoiu A, Dumitrescu D. Imaging tests for accurate diagnosis of acute biliary pancreatitis. World J Gastroenterol. 2014;20(44):16544-9. Doi: https://doi.org/10.3748/wjg. v20.i44.16544

17. International workshop on the clinical impact of endoscopic ultrasound in Gastroenterology. Working Party Report. Endoscopy. 2000;32:549-84. 